\title{
A biomechanical field testing approach in snow sports: Case studies toward a detailed analysis of joint loading
}

Proc IMechE Part P:

J Sports Engineering and Technology 2020, Vol. 234(4) 337-346

(c) IMechE 2020

Article reuse guidelines:

sagepub.com/journals-permissions DOI: $10.1177 / 1754337 / 20918037$

journals.sagepub.com/home/pip

(S)AGE

\author{
Nicolas Kurpiers' ${ }^{1}$, Paul McAlpine ${ }^{2}$ and Uwe G Kersting ${ }^{3,4}$
}

\begin{abstract}
In this study, kinematic and kinetic measurements were combined to assess the effects of removing the stiff shaft from a ski boot. It was hypothesized that joint flexion at the ankle, knee and hip increase and reduce joint loading specifically at the knee. A previously developed force sensor was combined with a high-speed camera system for data collection of 6 degrees of freedom ground reaction forces and three-dimensional marker data in the field on a wave slope. The collected data were used as input to a musculoskeletal model for the estimation of joint kinematics and joint moments and contact forces in the ankle and knee. The force sensor, which was previously used for skiing, had experienced wear and tear and was thus prone to breakage. As a result, joint loading could only be analyzed for two skiers. These two skiers did not use the added range of ankle flexion to its full extent, but showed substantial reductions in joint moments and joint contact forces (e.g. knee compression force from 85 to $57 \mathrm{~N} / \mathrm{kg}$ ). Only one of the five experienced skiers tested was able to adopt the anticipated movement pattern by substantially increased maximum ankle joint flexion angle (from $10^{\circ}$ to $37^{\circ}$ ) and knee joint flexion angle (from $93^{\circ}$ to $105^{\circ}$ ) and the respective ranges of motion when skiing through a wave course. The study provides information on possible individual adaptations to ski boot modifications. The mechanical construction of the force sensor will need to be modified to withstand the high forces expected during freestyle skiing. The study also supports the future use of this measurement setup for comprehensive studies in snow sports, provided that a sufficient training period is given.
\end{abstract}

\section{Keywords}

Freestyle skiing, ground reaction forces, joint loading, computer modeling, ski boot

\section{Introduction}

Freestyle skiing is a growing discipline in young people and has been associated with a comparably high risk of knee injuries. ${ }^{1}$ The total prevalence of injuries in alpine skiing is approximately two to three injuries per 1000 skier days ${ }^{2}$ and has decreased over the decades. However, severe knee injuries have tripled since $1970 .{ }^{3}$ In freestyle skiing, epidemiological data are only available from competitions which show the knee to be involved in approximately $50 \%$ of all injuries. In comparison, handball players show a lower incidence of knee injuries with approximately $11 \%$, where a comparison of exposure time remains difficult due to fundamental organizational differences of these sports. ${ }^{4}$ The indication of knee injury prevalence according to sports differs remarkably depending on the reference. Thus, basketball indications range from approximately $8 \%{ }^{5}$ to $33 \%{ }^{6}{ }^{6}$ while soccer indications range from $15 \%^{7}$ to more than $35 \%{ }^{8}$
In freestyle mogul skiing, knee injuries are predominant and can have devastating consequences for the skier. ${ }^{9}$ Previously, a ski boot modification was suggested to allow for a more natural, combined knee and ankle flexion, like in a squat movement without ski boots, to better negotiate bumps and potentially reduce the load on the knee joint. ${ }^{10}$ A previous study assessed the effect of ski boot hardness on wave course skiing

\footnotetext{
'Department of Sport Science, University of Hildesheim, Hildesheim, Germany

${ }^{2}$ High Performance Sport New Zealand, Auckland, New Zealand

${ }^{3}$ Institute of Biomechanics and Orthopaedics, German Sport University Cologne, Cologne, Germany

${ }^{4}$ Sport Sciences, Department of Health Science and Technology, Aalborg University, Aalborg, Denmark

\section{Corresponding author:}

Nicolas Kurpiers, Department of Sport Science, University of Hildesheim, Universitätsplatz I, W-4 I I, 3 I I I I Hildesheim, Germany.

Email: kurpiers@uni-hildesheim.de
} 
by a two-dimensional video approach, but found no alterations for commercially available soft and hard boots. It has been concluded that the range of ski boot hardness is not sufficiently large to allow for kinematic alterations. ${ }^{11}$ A different intervention, where the whole shaft of the ski boot was removed, has previously been utilized for balance training in competitive alpine skiers. This particular kind of boot intervention needs to be conceived as a coordination training tool, rather than an applicable modification, for competition or regular use. However, it may be considered as a simple modification and may be used in a concept validation experiment.

It is vital to assess training methods or equipment interventions in order to reduce the number of injuries in specific sports. While this has been done in indoor sports where potentially risky situations can be matched in a laboratory setup, ${ }^{12,13}$ the situation becomes more challenging in winter sports. In particular, the required attire of the participants to perform under low temperatures is making sensor and marker attachments a challenge. Previously, only a few investigations have measured kinematics in freestyle skiing, ${ }^{14,15}$ whereas others have included force and pressure measurements in skiing and freestyle skiing. ${ }^{10,16}$ Studies where kinematic and kinetic data sets were combined and used to calculate joint loading in skiing are still rare, ${ }^{17}$ while such an approach is required to fully investigate and understand injury mechanisms, joint loading and the effects of specific alterations to skiing equipment, in particular. Establishing a protocol in regard to snow sport, a quantitative analysis of freestyle-specific movements with specifically matched measurement equipment will allow for investigations of mechanical loading experienced by athletes during freestyle skiing.

The purpose of this study was twofold. First, the study was aimed at implementing a methodological approach combining video-based, three-dimensional (3D) movement data with kinetic data from a force sensor mounted between the ski and boot. These data were then imported into a musculoskeletal modeling system to estimate knee joint moments and joint contact forces. Second, the mentioned ski boot modification, with and without the shaft attached to the boot, was compared to verify that this approach is suitable to identify equipment-related changes in knee joint loading during skiing on a wave course. Accordingly, the first hypothesis was that the chosen measurement components were suitable for application to mogul skiing for training purposes. Mogul skiing versus skiing on a wave slope in this study is different as the latter does not include turns. However, the bending and straightening movements of the legs are the major component of mogul skiing that is restricted by the stiff ski boot shaft, forcing the skier into a potentially so-called "backseat position." This part of the complex movement can be assessed in a wave slope under simplified conditions, that is, turns excluded and an extreme modification. This extreme modification, as adopted from alpine ski racing, was used to assess the possibility of altering the motion patterns. The modification would be implemented in a less extreme nature for follow-up investigations in a mogul course including turns in order to approximate the actual mogul skiing technique and avoid harm to the ankle joints. Therefore, the second hypothesis was that the flexible (no shaft) boot would allow for an increased knee and ankle flexion on a wave course. The third hypothesis was that this change in technique would be accompanied by a reduced knee joint loading.

\section{Method}

Five male skiers $(27.8 \pm 11.9$ years $)$ were recruited for this study with their skill level ranging from "advanced recreational" to "expert." Ethical approval was obtained from the University of Auckland, New Zealand. Participants were interviewed about their technical skills, competitive level and training status prior to participation. They had to be injury free for at least 2 months prior to the study. Subsequently, they were introduced to the training boot and signed informed consent if they agreed to engage in the study. All testing took place during the fall on the Zermatt ski field, Switzerland, at an altitude of approximately $3500 \mathrm{~m}$ above sea level with temperatures ranging from $-15^{\circ} \mathrm{C}$ to $-5^{\circ} \mathrm{C}$.

Participants were asked to ski with the bindings adjusted to fit the test boots by an experienced ski service worker. They initially trained for at least $4 \mathrm{~h}$ with the new boots on the normal slope as well as on the wave course. They were instructed to practice bending and straightening movements in order to get accustomed to the altered flexibility of the boots and actively use the extended range of ankle movement, which is not possible with regular ski boots. The familiarization phase was conducted progressively, first with just the modified ski boot and, after $2 \mathrm{~h}$, with both the new boot and a mock-up of the force plate ${ }^{18}$ to practice the testing situation.

The tests were carried out on a wave slope of an average inclination of $19^{\circ}$ with six bumps of approximately $0.5 \mathrm{~m}$ height situated at a distance of $4 \mathrm{~m}$ apart from each other. Bumps number three and four framed the measurement area in order to collect the data within a fluent motion cycle. Snow fell twice on the testing area between the testing days, such that reconstruction of the test slope was necessary. The visibility varied within each testing day from slightly foggy to sunny and the wind intensity changed as well. However, even though the environment changed between runs, only marginal changes in climate or snow conditions occurred during individual testing sessions, which lasted for approximately $1 \mathrm{~h}$.

For modification of the boot, the ski boot's shaft was removed and the residual material at the back of the boot was made more deformable by milling two $80 \mathrm{~mm}$ vertical cuts into the rear spoiler, resulting in a 


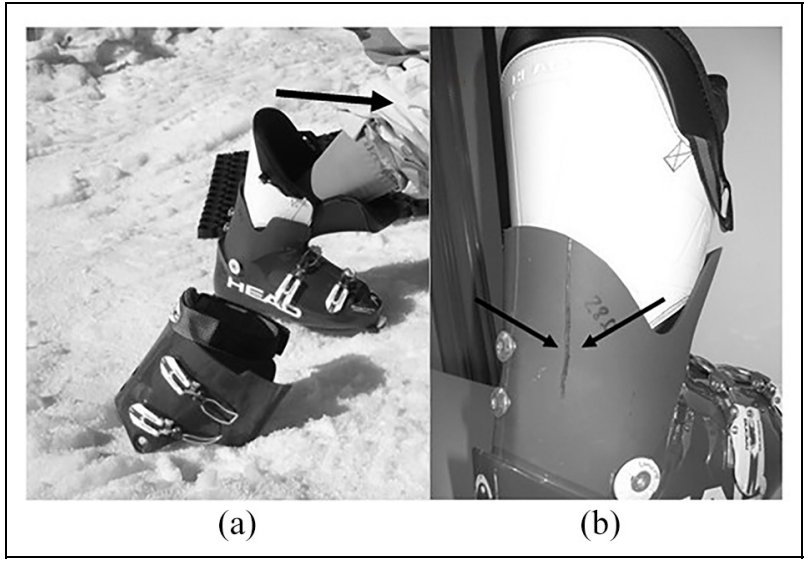

Figure I. Ski boot modification (Head Raptor Super Shape, Head and AUT): (a) greater flexibility in anterior direction and (b) $80 \mathrm{~mm}$ cut in rear spoiler to remove resistance in the posterior direction.

noticeably greater flexibility in both anterior and posterior directions (Figure 1). Three pairs of boots (Head Raptor Super Shape, Head and AUT) were made available for this study in sizes $28.0,28.5$ and $29.0 \mathrm{~cm}$.

For acquisition of kinetic data, two 6 degrees of freedom force sensors, developed specifically for skiing measurements, were utilized. ${ }^{19}$ The dynamometer parts were milled from aluminum, assembled and provided with strain gauges resulting in a weight of approximately $2 \mathrm{~kg}$ per piece, a height of $36 \mathrm{~mm}$ and a width of $62 \mathrm{~mm} .{ }^{19}$ Each sensor consisted of three different components, specifically the boot sole adapter, load cells and binding adapter (Figure 2). The ski boot was attached to the adapter with a buckle. Force data were amplified by custom-built strain-gauge amplifiers, sampled at $500 \mathrm{~Hz}, \mathrm{~A} / \mathrm{D}$ converted (model: USB4716, Advantech, USA) and stored onto a minicomputer using commercially available measurement software (Dasylab V.5; Measurement and Computing Corporation, USA). The coordinate system of the dynamometers was defined with the $\mathrm{x}$-axis anteroposterior (alongside the ski), y-axis medio-lateral (across the ski) and z-axis vertical to the ski. The midpoint of the sensor assembly was the origin of the associated coordinate system.

Four synchronized high-speed cameras (Basler A602f) and a Simi Motion System (Version 266; Simi Reality Motion Systems, Germany) were used to collect videos of the performance in a $10 \times 3 \mathrm{~m}$ area at a frequency of $100 \mathrm{~Hz}$. Two upper cameras and two lower cameras were situated aside the test slope on tripods, which stood in ice buckets to prevent any disruptive movements. The angle between any two optical axes was $60^{\circ}$ or more and the cameras had a distance of 7 $10 \mathrm{~m}$ to the recording volume.

A set of 27 black markers (19 dynamic and 8 static) were fixed to the racing suit of the skier bilaterally on bony landmarks to define the body parts (Table 1). A

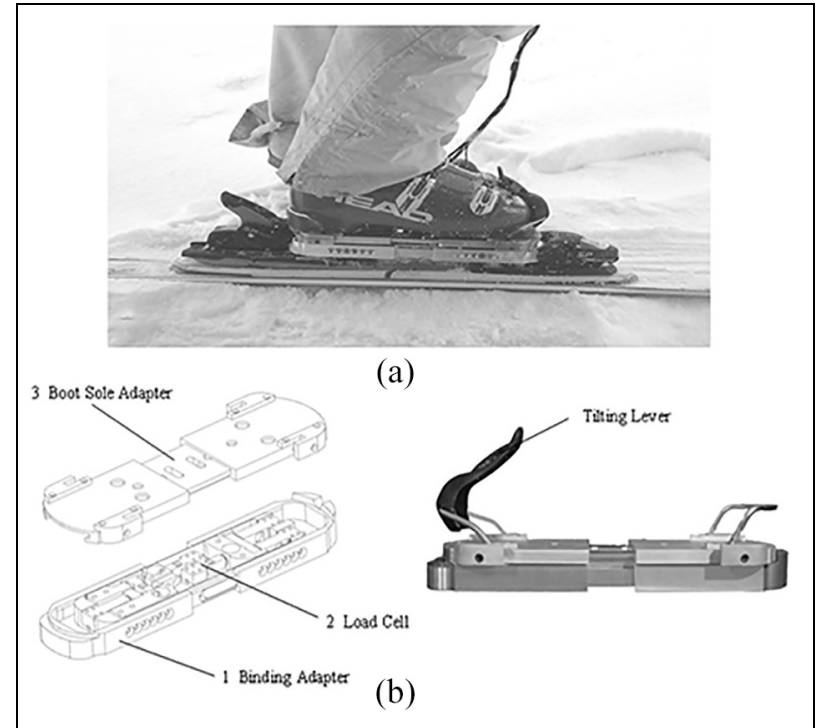

Figure 2. (a) Force sensor attached between ski boot and binding. (b) Three parts of the force sensor.

pole calibration method with a total of 36 calibration points was applied within the area of recording. Their positions were identified using a theodolite (Geodimeter ${ }^{\circledR}$ System 600, Germany) and entered into the Simi Motion System.

Participants were filmed during three runs wearing the modified flexible ski boot with no shaft (FL), performing bending and straightening movements in the wave slope, while ground reaction force (GRF) and associated moments were collected simultaneously. Subsequently, three runs with a standard ski boot (ST) were recorded. Synchronization of video and force recordings was managed off-line by including two short hops at the start of the test runs into the recordings.

All data were processed off-line for the kinematics using Simi Motion Software. The consecutive work steps in Simi Motion comprised the camera calibration, specification of the markers for digitization, the digitization itself and the calculation of the 3D marker coordinates using the direct linear transformation (DLT) method. ${ }^{20}$

Force data were converted using experimentally determined calibration factors $^{19}$ in a customized MATLAB (V.2013; The MathWorks, USA) routine, resampled, saved as text files and imported into the Simi Motion Software. For synchronization in Simi Motion, the reference hop visible in one of the cameras was used. A low-pass, recursive zero-time-lag secondorder Butterworth filter with a cut off-frequency of $10 \mathrm{~Hz}$ was applied to the kinematic data and $100 \mathrm{~Hz}$ for the force data. ${ }^{21}$

Subsequently, the preprocessed data served as the input to a musculoskeletal model. The AnyBody Modeling System (AnyBody Technology, Denmark) was used, which resolves joint moments in a first step and subsequently estimates muscle activations by 
Table I. Anatomical landmarks.

\begin{tabular}{|c|c|c|}
\hline Body segment & Marker name & Location \\
\hline Trunk & CLAV & $\begin{array}{l}\text { Cranial end of the } \\
\text { sternum }\end{array}$ \\
\hline \multirow[t]{4}{*}{ Hip } & RASI & $\begin{array}{l}\text { Right anterior superior } \\
\text { iliac spine }\end{array}$ \\
\hline & LASI & $\begin{array}{l}\text { Left anterior superior } \\
\text { iliac spine }\end{array}$ \\
\hline & RPSI & $\begin{array}{l}\text { Right posterior superior } \\
\text { iliac spine }\end{array}$ \\
\hline & LPSI & $\begin{array}{l}\text { Left posterior superior } \\
\text { iliac spine }\end{array}$ \\
\hline \multirow[t]{2}{*}{ Right thigh } & RTHIPROX & Right quadriceps proximal \\
\hline & RTHIDIST & Right quadriceps distal \\
\hline Left thigh & $\begin{array}{l}\text { LTHIPROX } \\
\text { LTHIDIST }\end{array}$ & $\begin{array}{l}\text { Left quadriceps proximal } \\
\text { Left quadriceps distal }\end{array}$ \\
\hline \multirow[t]{2}{*}{ Right knee } & RKNELAT & $\begin{array}{l}\text { Lateral epicondyle of } \\
\text { femur }\end{array}$ \\
\hline & RKNEMED & $\begin{array}{l}\text { Medial epicondyle of } \\
\text { femur (static) }\end{array}$ \\
\hline \multirow[t]{2}{*}{ Left knee } & LKNELAT & $\begin{array}{l}\text { Lateral epicondyle of } \\
\text { femur }\end{array}$ \\
\hline & LKNEMED & $\begin{array}{l}\text { Medial epicondyle of } \\
\text { femur (static) }\end{array}$ \\
\hline Right shank & RTIB & Medial to tibia \\
\hline Left shank & LTIB & Medial to tibia \\
\hline Right foot & $\begin{array}{l}\text { RTOE } \\
\text { RHEE } \\
\text { RANKLAT } \\
\text { RANKMED }\end{array}$ & $\begin{array}{l}\text { Second phalanges distales } \\
\text { Posterior to calcaneus } \\
\text { Lateral malleolus } \\
\text { Medial malleolus (static) }\end{array}$ \\
\hline \multirow[t]{2}{*}{ Left foot } & LTOE & Second phalanges distales \\
\hline & $\begin{array}{l}\text { LHEE } \\
\text { LANKLAT } \\
\text { LANKMED }\end{array}$ & $\begin{array}{l}\text { Posterior to calcaneus } \\
\text { Lateral malleolus } \\
\text { Medial malleolus (static) }\end{array}$ \\
\hline \multirow{2}{*}{$\begin{array}{l}\text { Equipment } \\
\text { markers }\end{array}$} & RFRONTBIN & Right front binding \\
\hline & $\begin{array}{l}\text { RREARBIN } \\
\text { LFRONTBIN } \\
\text { LREARBIN }\end{array}$ & $\begin{array}{l}\text { Right rear binding } \\
\text { Left front binding } \\
\text { Left rear binding }\end{array}$ \\
\hline
\end{tabular}

applying an optimization criterion; in this case, the overall muscle activation was to be minimized. The current body model is a modified full body model including the Twente lower extremity model, ${ }^{22}$ which has been adopted from the AnyBody Repository for the current application. Segment lengths were scaled to the marker data using the scaling algorithm proposed by Andersen et al. ${ }^{23}$

From the model output, the maximum and minimum ankle and knee flexion and extension angles, GRF and knee joint forces and moments were extracted. For this study, forces and moments at the knee joint were calculated using the local coordinate system of the tibia as it was common in other studies with $\mathrm{x}$-direction anteroposterior, $\mathrm{y}$-direction vertical and z-direction medio-lateral. ${ }^{24-26}$

The modified boot in this study was considered having no constraints to the ankle joint, whereas the normal boot did restrict the movement. The calculated ankle stiffness values for both conditions have been included in the "Anybody model" as an angledependent resisting moment at the ankle joint. Prior to this investigation, a separate boot stiffness test was conducted in order to gain specific ankle resistance stiffness for the stiff boot. ${ }^{27}$ This laboratory-based test was conducted using a phantom leg and foot, including a mechanical ankle joint in the boot attached to a rope with a force transducer and several markers. The applied load by pulling the rope, the displacement of the top of the prosthesis, as well as the moment arm of the load with respect to the ankle joint center were measured over the expected range of movement during skiing.

Only descriptive statistics were applied since only the data sets for two skiers included kinematic and kinetic results. In the graphs and the table, the means and standard deviations (SDs) over the three trials are presented.

\section{Results}

\section{Observations}

All participants felt challenged to ski freely on the slopes in the boot modification. When asked to perform test runs through the wave course, they managed without major problems, as it appeared easier for them to negotiate the waves. However, visual inspection revealed that the majority of the participants positioned themselves rather backward over the ski, contrary to the instructions given.

During the testing, the function and integrity of the binding sensors were thoroughly checked after each run. In several instances, metal connectors to the individual load cells were observed to be damaged or the function of the sensor was compromised by icing up. Overall, only two participants completed all test runs without sensor damage. Based on these design flaws, actions were taken to correct the issues following this experiment to provide a more robust construction for future studies.

\section{Kinematics}

The results show changes in joint angles consistently for the ankle joint. A greater maximum ankle joint flexion angle, as well as a greater ankle joint range of movement, was found when wearing the modified ski boot. The mean $(\mathrm{L} / \mathrm{R})$ maximum ankle dorsiflexion increased from $18^{\circ}$ to $14^{\circ}$ for skier 1 and $15^{\circ}$ to $3^{\circ}$ for skier 2 from the standard boot to the modified boot, respectively. The minimum dorsiflexion changed from $4^{\circ}$ to $-7^{\circ}$ and $-5^{\circ}$ to $-11^{\circ}$ for skier 1 and skier 2 , respectively. The resulting ranges of motion were increased for skier 1 , but decreased for skier 2. Both skiers were more dorsiflexed in their ankle joints in the stiff boot, but chose different ranges. Note that the angles reported here refer to the foot being flat on the ground in a neutral upright stance. In a ski boot, the foot points downward, which is accounted for in the model. 


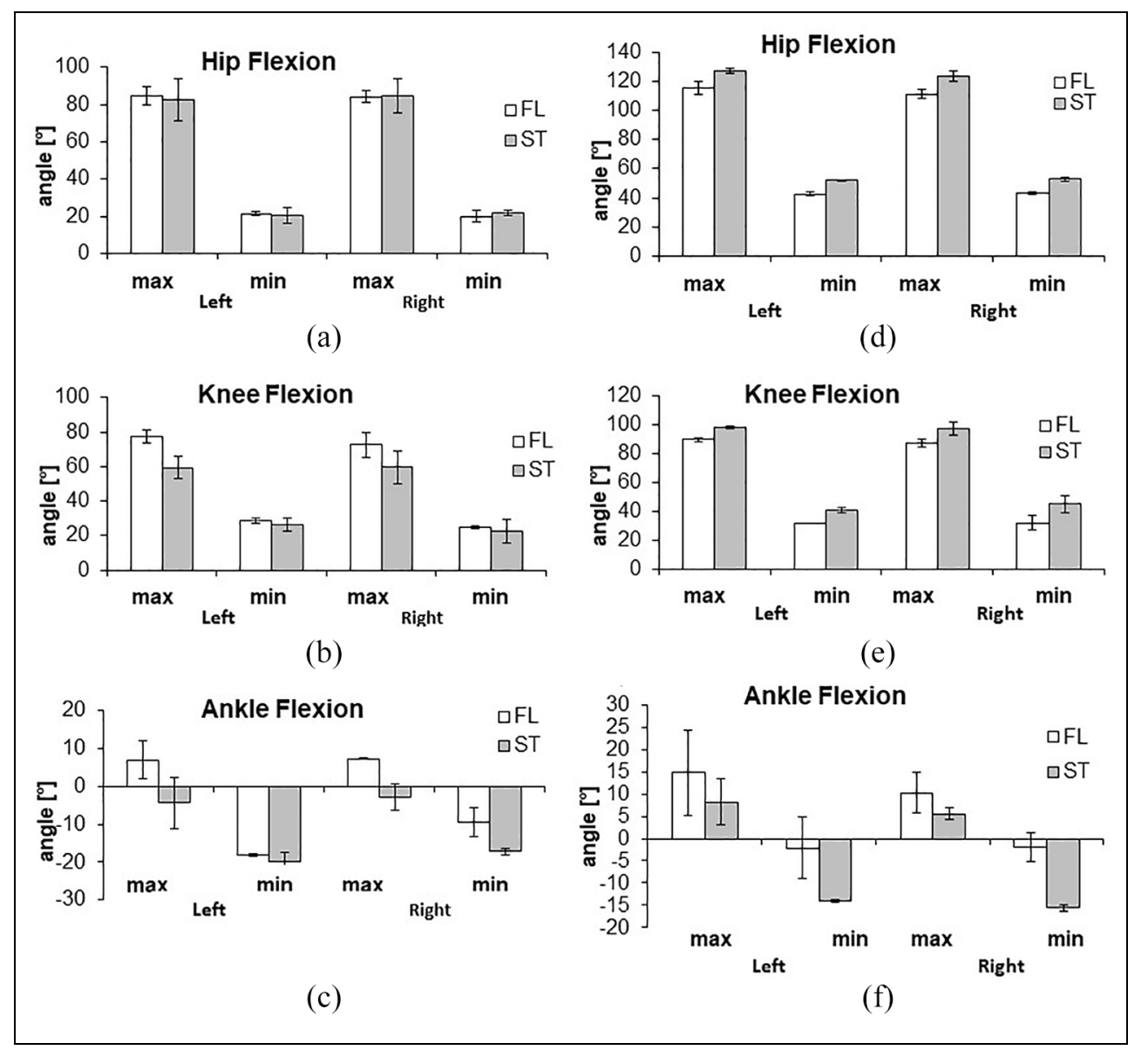

Figure 3. Kinematic results for skiers I and 2: (a) minimum and maximum hip flexion angles for the "flexible" (FL) and the "standard" (ST) condition (skier I); (b) minimum and maximum knee flexion angles (skier I); (c) minimum and maximum ankle flexion angles (skier I); (d) minimum and maximum hip flexion angles (skier 2); (e) minimum and maximum knee flexion angles (skier 2); and (f) minimum and maximum ankle flexion angles (skier 2).

In regard to knee flexion, a greater maximum flexion with only slightly increased minimum flexion was achieved by skier 1 . Therefore, he increased the range of motion in the knee joints by about $12^{\circ}$ from the standard to the modified condition, while keeping hip flexion unchanged. Skier 2 used slightly straighter knees and hips when skiing with the FL boot, while the ranges of motion remained virtually unchanged (Figure 3).

\section{Kinetics}

The magnitudes of the ankle joint moments were consistently reduced, while the minimum and maximum knee moments varied somewhat in direction and amount of change (Figure 4). The maximum normal binding forces were only slightly reduced. The knee compression forces applied to the tibial plateau were overall reduced by $20 \%$. The maximum force between patella and femoral condyle was generally smaller, resulting in a reduction of $30 \%$ on average (Table 2).

Ground reaction forces were reported in the local coordinate system of the ski force plate. Mean GRF and moments acting at the boot soles of both legs for two participants are displayed in Table 2 for both the FL and the ST conditions. The normal force $(\mathrm{Fz})$ is the main component of the resultant GRF, as the performance only comprised vertical movements of the legs. The GRF is approximately $1 \%$ larger in the ST condition with $10.1 \mathrm{~N} / \mathrm{kg}$ compared to $9.9 \mathrm{~N} / \mathrm{kg}$ in the FL condition for skier 1 , while the GRF is approximately $18 \%$ larger in the ST condition with $13.4 \mathrm{~N} / \mathrm{kg}$ compared to $10.9 \mathrm{~N} / \mathrm{kg}$ in the FL condition for skier 2 .

For the knee joint, an average reduction of maximum compression forces along the long axis of the tibia was shown, which was not symmetric and mainly affected the right knee. The minimum compression forces were symmetrical and on average reduced by $70 \%$ when comparing the FL boot to ST boot (Table 2). Finally, as the model contains a simplified knee mechanism, including the patella, an estimate was given for the force which the patella is experiencing when the quadriceps muscle is active. Apparently, this load varies from complete offloading to approximately $30 \mathrm{~N} / \mathrm{kg}$ for the FL boot on both legs, but increases by approximately $70 \%$ when skiing with the ST boot (Table 2). 
Table 2. Minimal and maximal force components for skiers I and 2.

\begin{tabular}{|c|c|c|c|c|c|c|c|c|}
\hline \multirow[t]{2}{*}{ Force component (N/kg) } & $\begin{array}{l}\text { Left } \\
\text { Skier I }\end{array}$ & SD & Right & SD & Left & SD & Right & SD \\
\hline & \multicolumn{4}{|l|}{ ST } & \multicolumn{4}{|l|}{$\mathrm{FL}$} \\
\hline $\begin{array}{l}\text { Normal force (min./max.) } \\
\text { Knee compression } \\
\text { force (min./max.) }\end{array}$ & $\begin{array}{l}0.3 / 8.5 \\
43.4 / 73.9\end{array}$ & $\begin{array}{l}1.8 / 0.5 \\
3.6 / 0.8\end{array}$ & $\begin{array}{l}2.3 / 11.7 \\
42.4 / 95\end{array}$ & $\begin{array}{l}0.3 / 0.6 \\
17.1 / 16.5\end{array}$ & $\begin{array}{l}1.7 / 9.3 \\
13.3 / 90.8\end{array}$ & $\begin{array}{l}0.1 / 0.5 \\
1.8 / 14.2\end{array}$ & $\begin{array}{l}2 / 10.6 \\
9.6 / 63.5\end{array}$ & $\begin{array}{l}0.4 / 0.5 \\
2.8 / 5.9\end{array}$ \\
\hline \multirow{3}{*}{$\begin{array}{l}\text { Patella compression } \\
\text { force ( } \min . / \max .)\end{array}$} & $0.1 / 15.1$ & $0.1 / 6.2$ & $0.4 / 11.3$ & $0.6 / 3.5$ & $0 / 9.5$ & $0 / 0.1$ & $0 / 11.8$ & $0 / 1.1$ \\
\hline & \multicolumn{8}{|l|}{ Skier 2} \\
\hline & \multicolumn{4}{|l|}{ ST } & \multicolumn{4}{|l|}{ FL } \\
\hline Normal force (min./max.) & $4.3 / 14.5$ & $1.4 / 3.8$ & $1.4 / 12.4$ & $0.5 / 0.6$ & $1.3 / 12.1$ & $0.5 / 3.5$ & $1.3 / 9.7$ & $0.1 / 1.9$ \\
\hline $\begin{array}{l}\text { Knee compression } \\
\text { force (min./max.) }\end{array}$ & $24.7 / 75$ & I8.5/6.| & $18 / 95.9$ & $3.5 / 16.5$ & $6.7 / 56.8$ & $2.4 / 8.7$ & $5.4 / 57.3$ & $0.2 / 7.8$ \\
\hline $\begin{array}{l}\text { Patella compression } \\
\text { force (min./max.) }\end{array}$ & $2.7 / 68$ & $0.7 / / 5.5$ & $2.8 / 36.8$ & $0.6 / 7.5$ & $0.1 / 36.4$ & $0.1 / 11$ & $0 / 23.8$ & $0 / 6.8$ \\
\hline
\end{tabular}

ST: standard; FL: flexible; SD: standard deviation.

Table 3. Mean values kinematics (angles in degrees, $n=5$ ).

\begin{tabular}{|c|c|c|c|c|c|c|c|c|}
\hline & \multicolumn{4}{|l|}{ ST } & \multicolumn{4}{|l|}{$\mathrm{FL}$} \\
\hline & Left & SD & Right & SD & Left & SD & Right & SD \\
\hline Hip flexion (min./max.) & $40 / 107.3$ & $12.6 / 17.4$ & $40.2 / 106.8$ & $12.4 / 16.8$ & $38 / 102.4$ & $10.2 / 12$ & $39.2 / 100.6$ & $12 / 12.6$ \\
\hline Knee flexion (min./max.) & $31.9 / 78.7$ & $9.8 / 21.2$ & $36.8 / 83.6$ & $10.4 / 18.3$ & $31.5 / 78.7$ & $4 / 17.9$ & $36.9 / 85.6$ & $6.3 / 11.1$ \\
\hline Ankle flexion (min./max.) & $-7 / 6.6$ & $7.5 / 6.2$ & $-7.6 / 11.8$ & $8.1 / 10.3$ & $-4.8 / 17.7$ & $9.2 / 12.9$ & $0.2 / 19.9$ & $6.8 / 12.8$ \\
\hline
\end{tabular}

ST: standard; FL: flexible; SD: standard deviation.

\section{Discussion}

This study had a twofold aim: exploring that a full 3D inverse dynamics analysis with the given testing devices is possible and illustrating this by assessing the effects of a ski boot intervention in a wave course. Five participants were tested (Table 3), but only two full data sets could be analyzed due to issues with connectors to the load cells within the force sensor assembly, resulting in unrecorded data. ${ }^{19}$ Thus, results from only two cases are presented here. For these two participants, comparably small kinematic changes were shown, except for the ankle joint with a $9^{\circ}$ increase in flexion on average. Kinetic changes were substantial at not only the ankle joints with up to $500 \%$ increase in the ankle moments on one side (Figure 4) but also affecting the knee with up to $70 \%$ increase on one side, even though the boot modification primarily altered the ankle movement. For comparison, Liebl et al. ${ }^{28}$ found ankle moments of approximately $2 \mathrm{Nm} / \mathrm{kg}$ in runners and Funken et al. ${ }^{29}$ assessed knee moments of approximately $4.6 \mathrm{Nm} / \mathrm{kg}$ in long jump. It may be noted that the gastrocnemius muscle activation increased up to $1100 \%$ in the ST condition, which suggests a strong correlation to the kinetic changes and thus the boot modification. The underlying idea of this boot alteration as a training tool was to allow for a natural squatting movement when flexing the legs to absorb a mogul and simultaneously reduce the joint loading on the knee, which only was the case in skier 2.

The increase in ankle flexion for the two skiers changed by $11^{\circ}$ and $3^{\circ}$, which indicates that these two subjects adapted to the ski boots by increasing both the maximum ankle joint flexion angle as well as the range of movement, whereas the knee joint angles only showed marginal changes. A study on a task similar to the current project reported comparable results; however, some differences were also observed. ${ }^{11}$ The authors tested a hard ski boot compared to a soft ski boot, which were both commercially available. The task for the testing procedure was the same as in this study, specifically skiing down a wave slope without turns absorbing the bumps. The study found minimal differences in joint angles between shoe types. In view of the current results, it can be proposed that substantial changes in boot hardness are associated with dramatic alterations to joint loading. The knee angles (KAs) were greater in this study in the extension directions but not in flexion. This could be explained by possible differences in the initial joint angle definitions, since the ranges of movement were similar in both studies. 


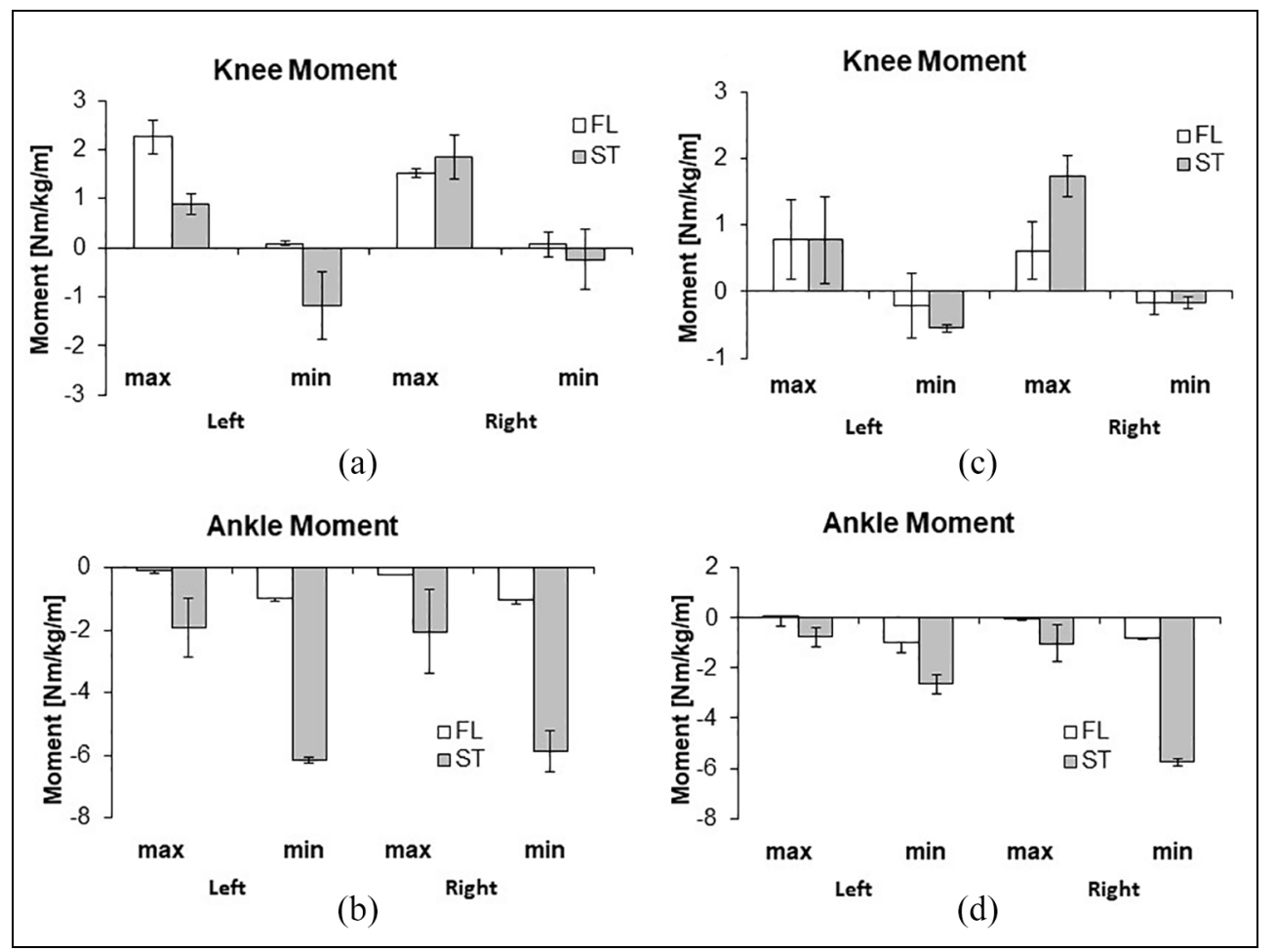

Figure 4. Selected kinetic results: (a) minimum and maximum knee moments for the FL and ST conditions (skier I); (b) minimum and maximum ankle moments for the FL and ST conditions (skier I); (c) minimum and maximum knee moments for the FL and ST conditions (skier 2); and (d) minimum and maximum ankle moments for the FL and ST conditions (skier 2).

It has to be noted that the kinematic results for the ankle joint in the FL condition appear to be slightly asymmetric and more variable for the FL boot (Figure 3(c)), which may be an effect of an uneven construction of the wave course. Due to the fact that the ski is comparably loosely linked to the leg in the FL condition, small variations in curvature of the snow surface may have generated this kinematic difference. While knee and hip joint kinematics remain relatively unchanged and symmetric, it is possible that this slight alteration has a substantial effect on the symmetry of muscle and joint loading parameters reported in this study. This is of particular interest as the future intention will be to investigate asymmetric tasks; however, in this experiment, only movements in the sagittal plane were expected. Such variations may be substantial, but unavoidable in field studies such as this one.

Despite the substantial alterations in joint loading and muscle activations, quite a large inter-individual variation was observed. This leads to the suspicion that the training period was likely insufficient for most participants to make full use of the greater forward flexibility of the boot, resulting in a different technique. Most participants, including the two presented here, skied in a fashion very similar to their conventional movement pattern. However, the training period with the modified equipment turned out to be vital for representative results because the greater dorsiflexion and less stability required adaptation to a different orientation on the skis. Neurophysiological studies indicate that changing the engram which leads to the execution of a learned "movement program" 30 is challenging to alter, depending on the difficulty level of the task. In the case of highly automatized and complex movements, a sufficient training period is required to establish a new movement strategy. ${ }^{31}$ In mogul skiing-related movements, a period of 3 days for familiarization has been shown to be useful to get accustomed to a different stance on the ski. ${ }^{32}$

In this study, only one participant was able to engage in such an extended training period due to time limitations and changeable weather conditions. Visual inspection revealed which participants showed a greater adaptation with an increased maximum ankle angle (AA) of $37^{\circ}$ in the FL condition compared to $10^{\circ}$ in the ST condition. Furthermore, a two times greater range of movement in the ankle joint was exhibited, corresponding to up to $10^{\circ}$ greater knee joint range of motion, as well as maximum angle and a more distinct forward lean indicated by a $13 \mathrm{~cm}$ more anterior projection of the center of the hip/mass with respect to a point halfway between the ankle joints ${ }^{18}$ (Figure 5). The change in maximum knee range of motion suggests a more active vertical absorbing movement with the modified boot, which is crucial for mogul skiing technique. However, as no kinetic data were collected for this subject, no implications for the resulting changes in joint loading can be made.

A strict standardization for the training period was not considered crucial as the major goal of this study 


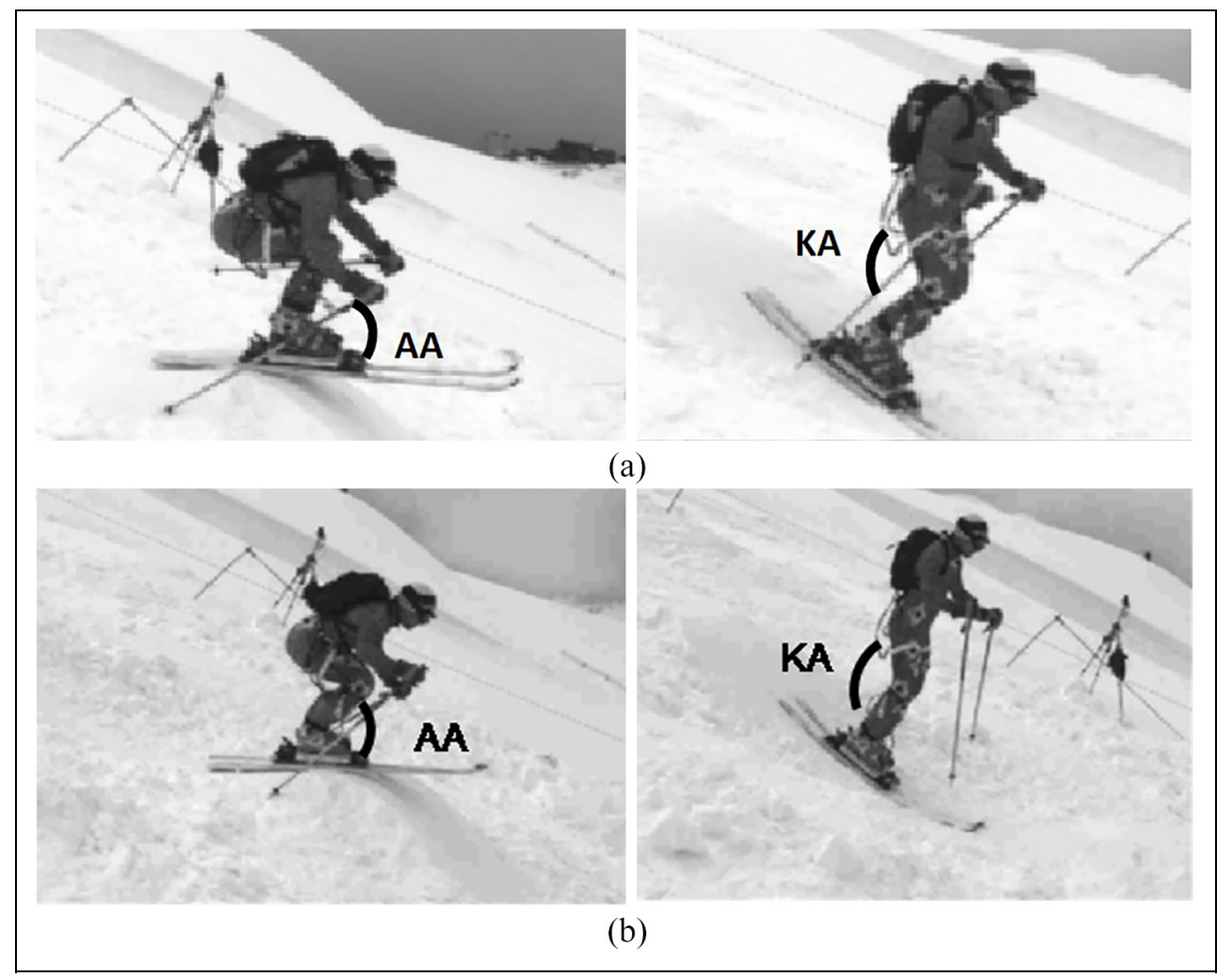

Figure 5. Test runs of participant 4 after three full days of familiarization with leg flexion on the bump and leg extension in the valley between bumps: (a) standard ski boot with shaft (ST) and (b) modified ski boot with no shaft (FL) (AA: ankle angle and KA: knee angle).

was the verification of the method. However, this aspect was considered vital for the implementation of followup studies. Participant 4 (Figure 5) was the only participant who trained for a full period of 3 days with the modified exercise boot. This is presumably reflected in the results that differ discernably in an intra-subject as well as in an inter-subject comparison. A greater anteriorly directed ankle range of movement may have caused the forward shifted center of mass during the test runs in the FL condition. This may have caused a reduction in the load on the passive structures of the knee. Such distinct changes were not found in other participants.

It is assumed that a lack of forward lean resistance led to a tendency to lean back at the instant of bump absorption to maintain balance and avoid falling. Thus, the boot modification may have been too drastic to gain a natural squatting movement above the base of support. No clear kinematic alterations were found for most participants and parameters; however, both participants showed a small, but consistent, decrease in peak GRF. Changes for joint forces and moments did not show a consistent pattern. A possible explanation is that the lower body kinematic strategy was adjusted by some participants in the way that an additional impact buffer was shared between the ankle, knee and hip joint almost evenly. Therefore, only slight changes to each joint in conjunction with a more active absorption by quickly pulling up the legs could theoretically have that effect. The knee compression forces support this explanation.

There are several limitations of this study. Differences in measurements can be encountered due to differences in the initial position of the model, the height of the moguls or the inclination of the testing slope. Regarding joint angles, some researchers determined zero in the static trial when the participant is already in a slightly flexed position and other researchers determined an upright position without boots as the $0^{\circ}$ or $180^{\circ}{ }^{33}$ Other aspects that lead to differences in the results of the two projects are camera adjustments, methods for digitization, accuracy of the force plates, calculation matrix for the GRF, choice of the model and model specifications. Several validation studies have been conducted on specific parameters used in the computer model for gait and cycling and showed good reliability of the current model predictions. ${ }^{34}$

The methodological differences need to be considered for the interpretation of the current results and results, in general. The movement task was simplified in this study to facilitate its execution within a short training period, but failed in four out of five participants. Performance in a wave slope is related to the vertical movements of the legs during mogul skiing. However, as mogul skiing includes turns, there are presumably different joint motions and loads transferred 
to the knee joint, which need to be investigated in follow-up studies, including turns. The varying results of this study can be explained by the lack of training time and changes in stability of the boot intervention.

\section{Conclusion}

In conclusion, this study successfully verified the testing process, including motion analysis in an outdoor skiing environment, a rather drastic boot modification and the utilization of the computer model. This conclusion is valid, provided a further improvement loop of the system guarantees the avoidance of icing of the load cells and the damage of specific parts of the force sensors by reinforcement. By the time this article is published, this requirement had been met. The removal of the shaft impedes the adjustments of joint movements by changes in muscle activity if no sufficient training time is given. In this study, only one participant managed to kinematically make full use of the boot alteration. In the future, this specific training tool should still be considered for several skiing disciplines on different skill levels; however, caution should be used as ankle protection by the boot is not maintained. Hence, further data collection is required with sufficient training time and an improved ski boot modification in followup studies.

\section{Acknowledgements}

We want to thank Zermatt Bergbahnen AG, Jürg Biner, Veit Senner, Andreas Kiefmann, Peter Spitzenpfeil and all participants.

\section{Declaration of conflicting interests}

The author(s) declared no potential conflicts of interest with respect to the research, authorship, and/or publication of this article.

\section{Funding}

The author(s) received no financial support for the research, authorship, and/or publication of this article.

\section{ORCID iD}

Nicolas Kurpiers (iD https://orcid.org/0000-0002-36225282

\section{References}

1. Krosshaug T. Validation of a model based imagematching technique for three-dimensional motion reconstruction from uncalibrated two-dimensional video sequences (Abstract). Knee Surg Sports Traumatol Arthros 2002; 10(6): 387-388.

2. Koehle MS, Lloyd SR and Taunton JE. Alpine ski injuries and their prevention (Les traumatismes en ski alpin et la prevention.). Sports Med 2002; 32(12): 785-793.
3. Hunter RE. Skiing injuries. Am J Sports Med 1999; 27(3): 381-389.

4. Lian ØB, Engebretsen L and Bahr R. Prevalence of jumper's knee among elite athletes from different sports: a cross-sectional study. Am J Sports Med 2005; 33(4): 561-567.

5. Ellison L. Basketball injuries in the database of the Canadian Hospitals Injury Reporting and Prevention Program (CHIRPP). Chronic Dis Can 1995; 16(3): 117-124.

6. Louw Q, Grimmer K and Vaughan K. Knee injury patterns among young basketball players in Cape Town. $S A$ $J$ Sports Med 2003; 15(1): 9-15.

7. Zillmer DA, Powell JW and Albright JP. Gender-specific injury patterns in high school varsity basketball. Journal of Women's Health 1992; 1(1): 69-76.

8. Hickey GJ, Fricker PA and McDonald WA. Injuries of young elite female basketball players over a six-year period. Clin J Sport Med 1997; 7(4): 252-256.

9. Heir S, Krosshaug T and Ekeland A. The incidence and trends of ACL injuries in world cup freestyle skiing during a 10 year period. Br J Sports Med 2005; 39(6): 389.

10. Schaff $\mathbf{P}$ and Hauser W. Influence of ski boot construction on knee load: a biomechanical investigation on safety and performance aspects of ski boots. In: Johnson RJ, Mote CD Jr and Celcer J (eds) Skiing trauma and safety: ninth international symposium. Philadelphia, PA: ASTM, 1993. pp.75-88.

11. Machens P. Einfluss eines neuen Konzepts alpiner Skischuhe auf das muskulaere Zusammenspiel im Buckelpisten-Skilauf. Munich: University of Technology, 2006.

12. McLean SG, Fellin R, Suedekum N, et al. Impact of fatigue on gender-based high-risk landing strategies. Med Sci Sports Exer 2007; 39(3): 502-514.

13. Zebis MK, Andersen LL, Bencke J, et al. Identification of athletes at future risk of anterior cruciate ligament ruptures by neuromuscular screening. Am J Sports Med 2009; 37(10): 1967-1973.

14. Arndt AN and Milburn PD. A three-dimensional kinematic analysis of alpine skiing in moguls. J Biomech 1994; 27(6): 653.

15. Krosshaug $\mathrm{T}$ and Bahr $\mathrm{R}$. A model based imagematching technique for $3 \mathrm{D}$ motion reconstruction from uncalibrated video sequences: application to WC freestyle skiing. Br J Sports Med 2005; 39(6): 394.

16. Klous M, Mueller E and Schwameder H. Knee joint loading in alpine skiing: a comparison between carved and skidded turns. In: Proceedings of the 12th annual congress of the European College of Sport Science: book of abstracts, Jyvaskyla, 11-14 July 2007, pp.99-100.

17. Lehner S. Entwicklung und Validierung biomechanischer Computermodelle und deren Einsatz in der Sportwissenschaft. Koblenz: Universitaet Koblenz-Landau, 2007.

18. Kurpiers N, McAlpine P and Kersting UG. Perspectives for comprehensive biomechanical analyses in mogul skiing. Res Sports Med 2009; 17(4): 231-244.

19. Kiefmann A, Krinninger M, Lindemann U, et al. A new six component dynamometer for measuring ground reaction forces in alpine skiing. Sports Engineering 2006; 9(3): 87-92.

20. Chen L, Armstrong CW and Raftopoulos DD. An investigation on the accuracy of three-dimensional space reconstruction using the direct linear transformation technique. J Biomech 1994; 27(4): 493-500. 
21. Corazza S and Cobelli C. An innovative ski-boot: design, numerical simulations and testing. J Sports Sci Med 2005; 4(3): 229-238.

22. Klein Horsman MD. The Twente lower extremity model: consistent dynamic simulation of the human locomotor apparatus. PhD Thesis, Universiteit Twente, Twente, 2007.

23. Andersen MS, Rasmussen $\mathbf{J}$ and Damsgaard M. Scaling and local marker coordinates determination of musculoskeletal systems. SAE technical paper 2007-01-2477, 2007.

24. Herzog W, Read L, Kerwin DG, et al. Anterior cruciate ligament forces in alpine skiing. J Appl Biomech 1993; 9: 260-278.

25. Read L and Herzog W. External loading at the knee joint for landing movements in alpine skiing. Int J Sport Biomech 1992; 8(1): 62-80.

26. Nachbauer W and Kaps P. A video technique for obtaining 3D coordinates in alpine skiing. J Appl Biomech 1996; 12(1): 104-115.

27. Kurpiers N. Dynamics of freestyle skiing: equipment development and implications of injury prevention strategies. PhD Thesis, The University of Auckland, Auckland, New Zealand, 2010.

28. Liebl D, Willwacher S, Hamill J, et al. Ankle plantarflexion strength in rearfoot and forefoot runners: a novel cluster analytic approach. Human Mov Sci 2014; 35: 104-120.

29. Funken J, Willwacher S, Heinrich K, et al. Long jump mechanics: Olympic versus Paralympic champion. ISBS P Arch 2017; 35(1): 151.

30. Monfils MH, Plautz EJ and Kleim JA. In search of the motor engram: motor map plasticity as a mechanism for encoding motor experience. Neuroscientist 2005; 11(5): 471-483.

31. Ericsson KA. Deliberate practice and the acquisition and maintenance of expert performance in medicine and related domains. Acad Med 2004; 79(10): 70-81.

32. Kurpiers N, Petrone N, Wisser A, et al. The effect of ski boot flexion stiffness on jump landings in skiing. In: Müller E, Kroell J, Lindinger S, et al. (eds) Science and skiing VII: 7. Maidenhead: Meyer \& Meyer, 2018, pp. 142-151.

33. McAlpine P. Development of a field testing protocol for the biomechanical analysis of snowboard jump landings. Master's Thesis, The University of Auckland, Auckland, New Zealand, 2006.

34. Dendorfer S and Toerholm S. Moment arm validation of the Twente lower extremity model. Aalborg: Institute of Mechanical Engineering, Aalborg University, 2008. 